\title{
A ROBOT CLIMBING VEHICLE AND ITS MAN-MACHINE INTERFACE FOR USE IN THE NUCLEAR INDUSTRY
}

\author{
T.S.White BEng; "A.A.Collie BSc, CEng, MIEE: \\ Prof J Billingsley MA(Cantab), PhD, CEng, FIEE; \\ B.L.Luk BSc, MSc; D.A.Moseley BEng. \\ School Of Systems Engineering, \\ Portsmouth Polytechnic, \\ Anglesea Building, \\ Anglesea Road, \\ Portsmouth, \\ England. \\ PO3 1DJ
}

\begin{abstract}
This paper describes the design and performance of a robot system NERO (Nuclear Electric's Robot Operator), commissioned by Nuclear Electric PLC. The robot was first operational in February 1991. The system consists of a robot climbing vehicle and a specially designed control console. The robot is pneumatically powered and uses eight vacuum sucker feet as grippers. The man-machine interface is designed to be easy to use and provides clear, concise status information, at all times maintaining safe reliable operation. At the time of writing the vehicle has successfully completed trials at Nuclear Electric test facilities.
\end{abstract}

\section{INTRODUCTION}

The use of mobile robots is now well established in the production areas of automated factories. The latest applications for automated vehicles are in hazardous and unpredictable environments of the Petro-Chemical, Construction and Nuclear Industries. In these applications the vehicle takes a tool to the workplace as apposed to taking the workpiece to the machine tool.

The robot system commissioned by Nuclear Electric was required for a specific application. Its associated control system and software were developed from those used on the previous Portech walkers and climbers. These factors allowed the robot to be developed from conception to a working vehicle in a period of seven months. The vehicle's basic application is as a remote inspection device with facilities for carrying payloads and additional manipulators. 


\section{VEHICLE DESIGN}

The mechanisms structure is constrained by the application and design specification. It is essentially simple and consists of two rectangular frames, an inner and an outer frame. Each frame carries its own set of four vacuum gripper feet on pneumatic 'leg' cylinders. A double rod, double acting cylinder is used as the frame translation actuator. The end of the rods are attached to the outer frame whilst the cylinders body is attached to a plate which is itself connected to the inner frame by a rotary joint. Rotary actuation is achieved by a further double acting cylinder which is mounted on the inner frame and linked to the sliding plate. To provide compliance with curved or uneven surfaces the gripper feet are attached to the frame by means of ball joints.

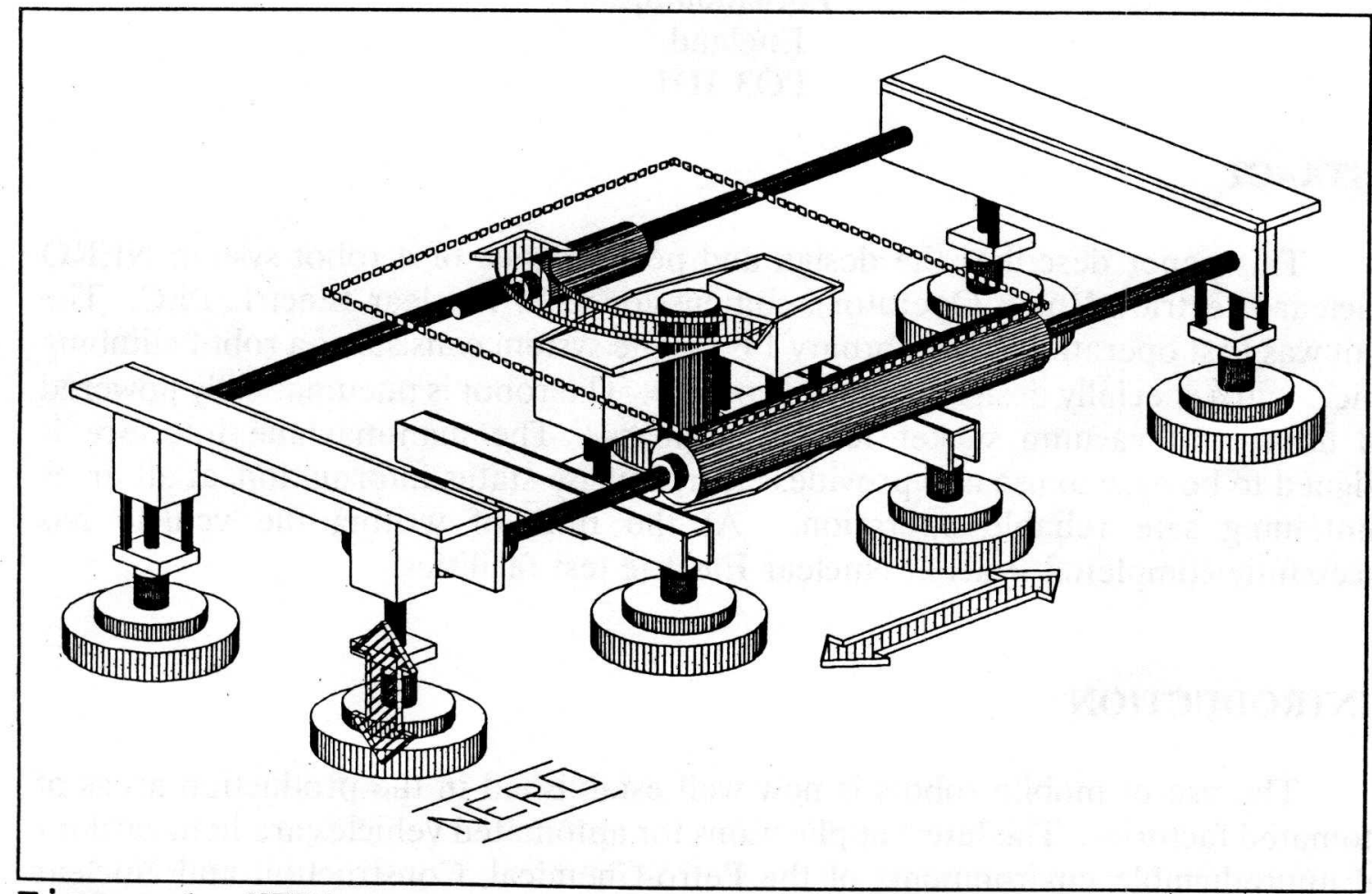

Figure 1 NERO

Motion is achieved by sequential stepping or rotating. The set of legs corresponding to one of the frames extend and its feet grip. The feet of the other frame do not activate and lift allowing either translation or rotation to be performed. Once the end stop of a cylinder is reached, movement in the same direction is achieved by swapping the raised frame with the gripping frame. The frame with gripping feet is swapped with the other via an all eight feet gripping stage.

The choice of pneumatic actuation gives the structure the high power to weight ratio essential for climbing [1], [2]. Vacuum gripper feet were chosen over flux switched magnetic feet because of the later's bulkiness and non-retrievability in one of the failure modes considered. The vacuum gripper design used [3] consists of an aluminium body housing a vacuum ejector pump and having a soft rubber seal. The foot is constantly supplied with air and can either 'suck' or 'blow'. The 'blowing' 
action not only has the effect of cleaning filters in the feet, but also clears loose material from under the foot as it is placed down, reducing contamination.

The vehicle is controlled by an on-board microprocessor unit. Power, a communication channel, video return and compressed air are supplied to the vehicle by means of an umbilical. The on-board microprocessor provides low level control for all aspects of the vehicle's motion. The microprocessor unit also has enough spare capacity for control of a simple manipulator or tool. The microprocessor and other electronics have been tested in radiation levels equivalent to being within the biological shield of a shut down reactor. The equipment was found to be sufficiently radiation resistant to work for considerable periods.

Solenoid valves activate the cylinders which are controlled by pressure and displacement signals fed back to the microprocessor unit. The method of control is essentially by discrete pulses with force or position feedback. The quality of vacuum is also fed back as a signal from a pressure sensor, ensuring that integrity of grip is maintained during the foot changeover sequence.

An important safety feature of the system is the effect of electrical power failure. Due to the arrangement of the control valves the vehicle stops and 'sucks' with all eight feet. This means that only a compressed air failure would cause the vehicle to fall from its climbing surface, and this failure mode is protected against by dual air supply.

Additionally the vehicle carries front and rear video cameras with auto-iris lens control. Associated with these cameras are two sets of lights of variable intensity. The vehicle surroundings can also be observed by segmented collision sensors attached to each of the feet.

\section{CONTROL CONSOLE AND SOFTWARE}

The vehicle is operated from a specially designed, self contained, control console. This unit contains a 80386 based IBM compatible microcomputer, a control panel and the power supplies required for the robot vehicle. The console requires only mains electricity and compressed air at $8 \mathrm{Bar}$. All other electrical and pneumatic interconnections take place within the console.

In the event of electric or pneumatic supply failures a number of safeguards have been included. If electrical failure occurs, an uninterruptible power supply switches in. The supply is capable of keeping both the computer and the vehicle (without lights) running for approximately 15 minutes. This allows enough time to backtrack the vehicle to a safe position and shut down the system, storing the vehicle status variables at the same time. Pneumatically the console has two safety features. An audio/visual alarm is triggered when the air pressure drops below a certain value, and two air compressors (and/or storage tanks) may be connected to the system at the same time. A valve automatically switches between these if necessary.

The control system is arranged in a hierarchical structure both in terms of hardware and in software. At the top of the system hierarchy is the microcomputer which serves as a supervisory computer. It performs path planning, on-line user 
interaction and maintains safe, reliable control of the vehicles actions. The top level computer monitors a specially designed control panel, interprets the users requirement and sends the appropriate commands to the on-board microprocessor. Communication is via an RS422 serial link through the umbilical.

The software also exhibits a hierarchical structure. The lowest level is written in machine code within the control unit of the crawler. This has been kept simple, lowering the risk, and minimising the effects, of a low level crash within the radioactive environment. The low level software provides open-loop control of the unit, the loop being closed by the high level computer. This is a deliberately slow but reliable method of control. The hierarchical structure continues in the 'master' computer. The top level being the user interface.

The user operates the system by either of two input devices, the computer keyboard or a specially designed control panel. Normally control is via the more ergonomically acceptable joy-sick on the control panel. The keyboard is locked out unless special functions are required.

\section{MAN-MACHINE INTERFACE}

The man-machine interface consists of the input devices described, the computer monitor and a video monitor. The interface as a whole is designed to be simple to use and to present an uncluttered representation of the robots status. This is important because the operators may be working under pressure.

The control panel shown in figure 2 contains all the necessary functions for operating the vehicle, its lights and cameras. the functions of the switches are described in table 1.

\begin{tabular}{||l|l|l||}
\hline No & Type & Function \\
\hline 1 & Switch & Power switch for robot. \\
\hline 2 & Switch & Power switch for lights. \\
\hline 3 & Key Lock & Switch to disable control panel, prevents unauthorised use. \\
\hline 4 & Key Lock & Switch to access keyboard control and advanced functions. \\
\hline 5 & Key Lock & $\begin{array}{l}\text { Switches cameras and lights. The joy stick is also effected, its } \\
\text { action is relative to the camera view. }\end{array}$ \\
\hline 6 & Neon Lamp & low air pressure warning, coupled to a buzzer. \\
\hline 7 & Button & Emergancy stop. \\
\hline 8 & Rotary & $\begin{array}{l}\text { Controls intensity of lights, also effects camera focus because of } \\
\text { auto-iris lenses. }\end{array}$ \\
\hline 9 & Button & $\begin{array}{l}\text { Commands the computer to move a frame to the center of } \\
\text { rotation. }\end{array}$ \\
\hline
\end{tabular}




\begin{tabular}{||l|l|l||}
\hline 10 & Button & $\begin{array}{l}\text { Commands the computer to move a frame to the center of } \\
\text { translation. }\end{array}$ \\
\hline 11 & Button & Confirms an action or initiates frame change. \\
\hline 12 & Switch & Selects 'part' to be acted on. \\
\hline 13 & Joy Stick & Proportional control for movements. \\
\hline 14 & Button & Fire button, activates joystick. \\
\hline 15 & Button & Concealed reset button. \\
\hline
\end{tabular}

Table 1. Control Panel Functions

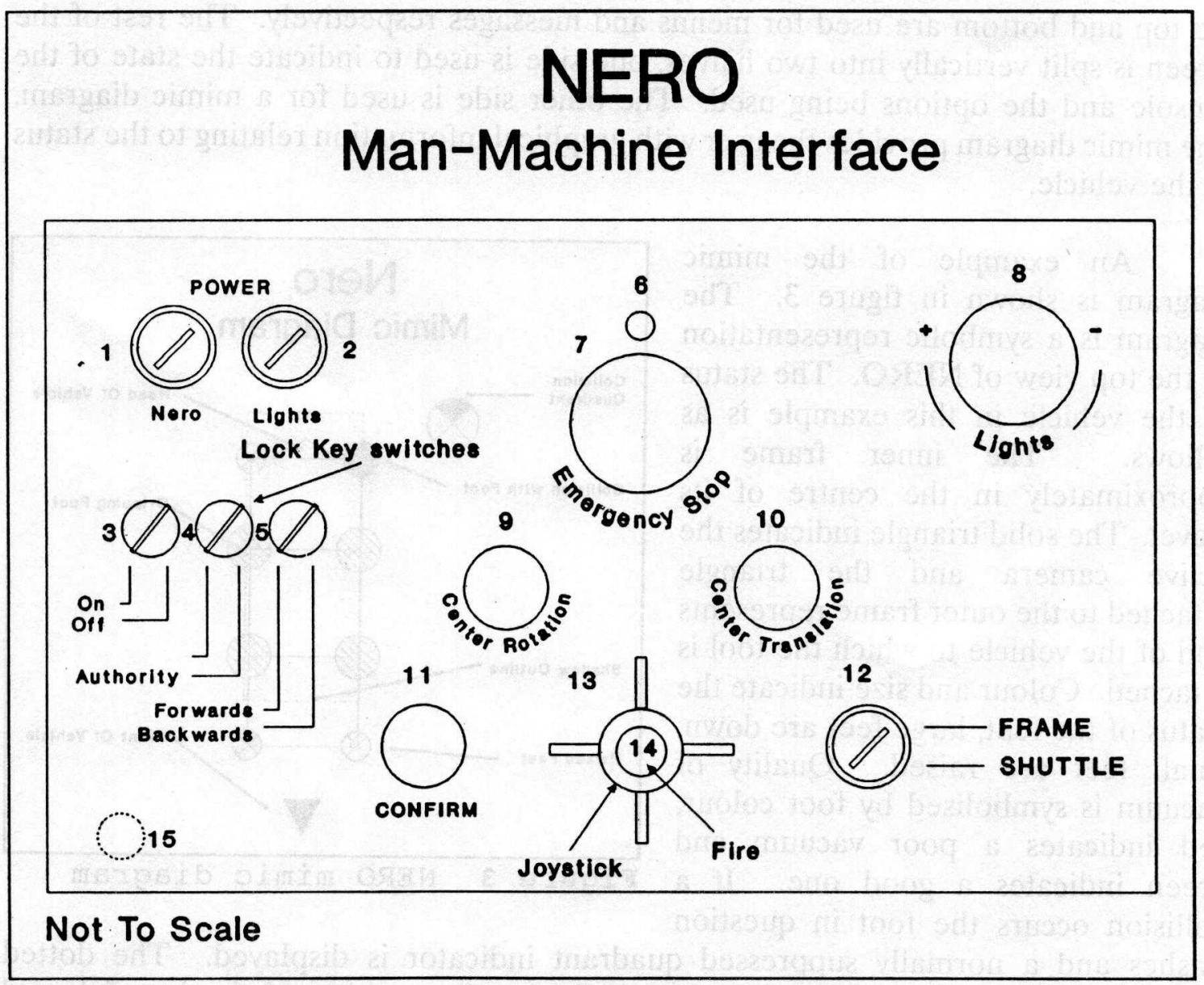

Figure 2 Control console panel

Basic movements of the vehicle are achieved by the following sequence. The joy stick is moved in the required direction, this has no effect until the 'fire' button is pressed, thus preventing accidental use. When the vehicle is in its required position the 'fire' button is released. To swap the gripping frame with the free frame, the 'confirm' button is pressed. This has the action of lowering the free frame and gripping with all eight feet. To free the other frame it is first selected by the 'part' switch (12) and then the 'confirm' button releases it. All actions the user makes are monitored by the computer, in the event of a 'dangerous' request, the command is 
disregarded and the user informed by error messages.

The vehicle may be operated adequately by the video monitor and the control panel alone, as many remotely operated vehicles are. However the use of the information provided on the computers screen enhances the users awareness of the vehicles status.

The visual interface via the computer provides a full description of the console's status, in an uncluttered format of indicators and icons. The data screen is kept simple by showing option menus only when requested by the 'authority' key. Status information such as collision detection or messages are suppressed from the screen until that data becomes active.

The normal information screen is split into four windows: small windows at the top and bottom are used for menus and messages respectively. The rest of the screen is split vertically into two halves, one side is used to indicate the state of the console and the options being used. The other side is used for a mimic diagram. The mimic diagram provides the user with graphical information relating to the status of the vehicle.

An example of the mimic diagram is shown in figure 3 . The diagram is a symbolic representation of the top view of NERO. The status of the vehicle in this example is as follows. The inner frame is approximately in the centre of its travel. The solid triangle indicates the active camera and the triangle attached to the outer frame represents end of the vehicle to which the tool is attached. Colour and size indicate the status of the feet, large feet are down, small feet are raised. Quality of vacuum is symbolised by foot colour, red indicates a poor vacuum and green indicates a good one. If a

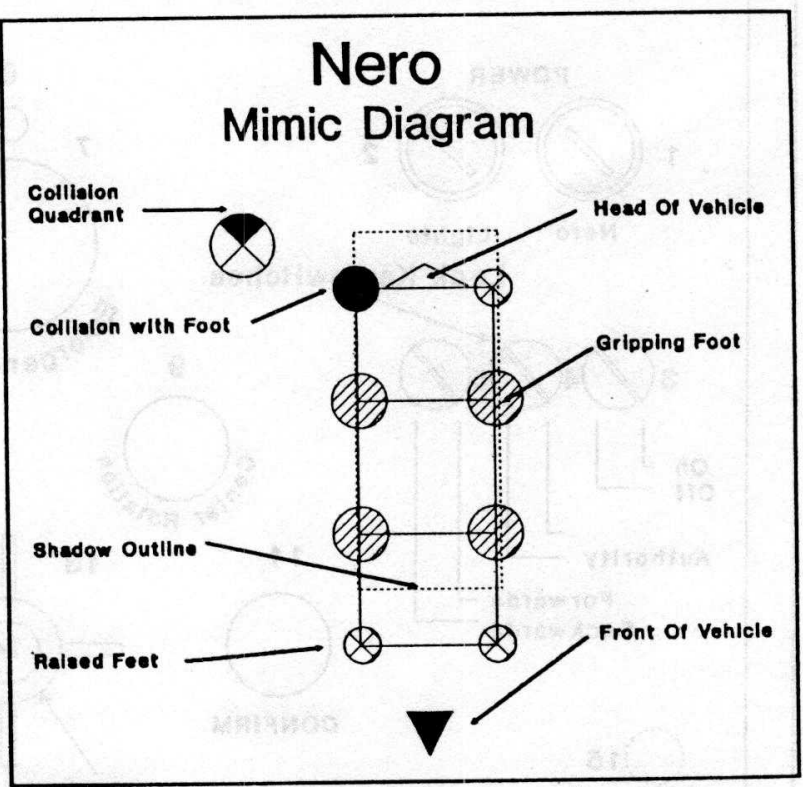

Figure 3 NERO mimic diagram collision occurs the foot in question flashes and a normally suppressed quadrant indicator is displayed. The dotted rectangle labelled as a shadow outline indicates the position of the joy-stick and hence where the vehicle is moving to. It should be noted that the joy-sticks action is relative to the cameras view, in this case it has been pulled back.

As has been stated earlier the man-machine interface has a number of other control options. These are made available via a menu when the manual 'authority' key is used, operators requiring such function seek permission and the key from their supervisor. The options available include calibration facilities, auxiliary manipulator control and path-back track functions. Facilities for interrogating and accepting foot vacuum quality and accepting activated collision sensors are also available. 


\section{PERFORMANCE}

The vehicle has undertaken several trials in scenarios which are similar to its designed application. At the time of writing all the trials have not been completed. However NERO has successfully climbed and traversed (with payloads) a vertical cylindrical surface, of rough steel, negotiating obstacles in its path. It can step over small obstacles of less than $25 \mathrm{~mm}$ in height.

The table below gives the vehicles specifications. Trials to date have shown that the vehicle performs to these parameters.

\begin{tabular}{|c|c|c|}
\hline & NERO SPECIFICATION & VALUE \\
\hline 1 & Length & $0.780 \mathrm{M}$ \\
\hline 2 & Width & $0.300 \mathrm{M}$ \\
\hline 3 & Height & $\begin{array}{l}0.131 \mathrm{M}-\mathrm{Max} \\
0.156 \mathrm{M}-\mathrm{Min}\end{array}$ \\
\hline 4 & Weight & $18 \mathrm{Kg}$ \\
\hline 5 & Maximum Stride & $0.200 \mathrm{M}$ \\
\hline 6 & Maximum Stepover Height & $0.025 \mathrm{M}$ \\
\hline 7 & $\begin{array}{c}\text { Speed } \\
\text { (Restricted Operational Speed) }\end{array}$ & 2 Metres/Minute \\
\hline 8 & Single Foot Holding Force & 300 Newtons \\
\hline 9 & $\begin{array}{l}\text { Maximum Payload } \\
\text { (Vertical Climb) }\end{array}$ & $15 \mathrm{Kg}$ \\
\hline 10 & $\begin{array}{c}\text { Air Consumption } \\
\text { (Supplied at } 10 \text { Bar and Restricted to } 8 \text { Bar at } \\
\text { Robot) }\end{array}$ & 240 Litres/Minute \\
\hline 11 & Cameras & $\begin{array}{l}\text { Front and Rear CCd Video } \\
\text { Cameras, Remotely Viewed }\end{array}$ \\
\hline 12 & Lighting & 600W Max Front or Rear \\
\hline 13 & Umbilical & $50 \mathrm{~m}$ \\
\hline 14 & Operating surface & $29 \mathrm{~m}$ diameter sphere \\
\hline
\end{tabular}

Table 2. Nero Specifications

\section{ACKNOWLEDGEMENTS}

The authors wish to thank Nuclear Electric PLC for permission to publish this paper and for their useful discussions during the development and vehicle trials. 


\section{REFERENCES}

[1] COLLIE A.A, BILLINGSLEY J, and HATLEY L, 'The Development of a Pneumatically Powered Walking Robot Base.', Proc ImechE conference C371/86.

[2] COLLIE A.A, BILLINGSLEY J, and Von PUTTKAMER E,' Design and Performance of the Portsmouth Climbing Robot.', Proc 7th International Symposium on Automation and Robotics in Construction.

[3] Subject of patent application.

[4] COLLIE A.A, CURRY A, 'A Climbing Vehicle for Nuclear Reactor Inspection.', Proc Conference on Remote Technology for the Nuclear Industry, 10-11-90 London. 


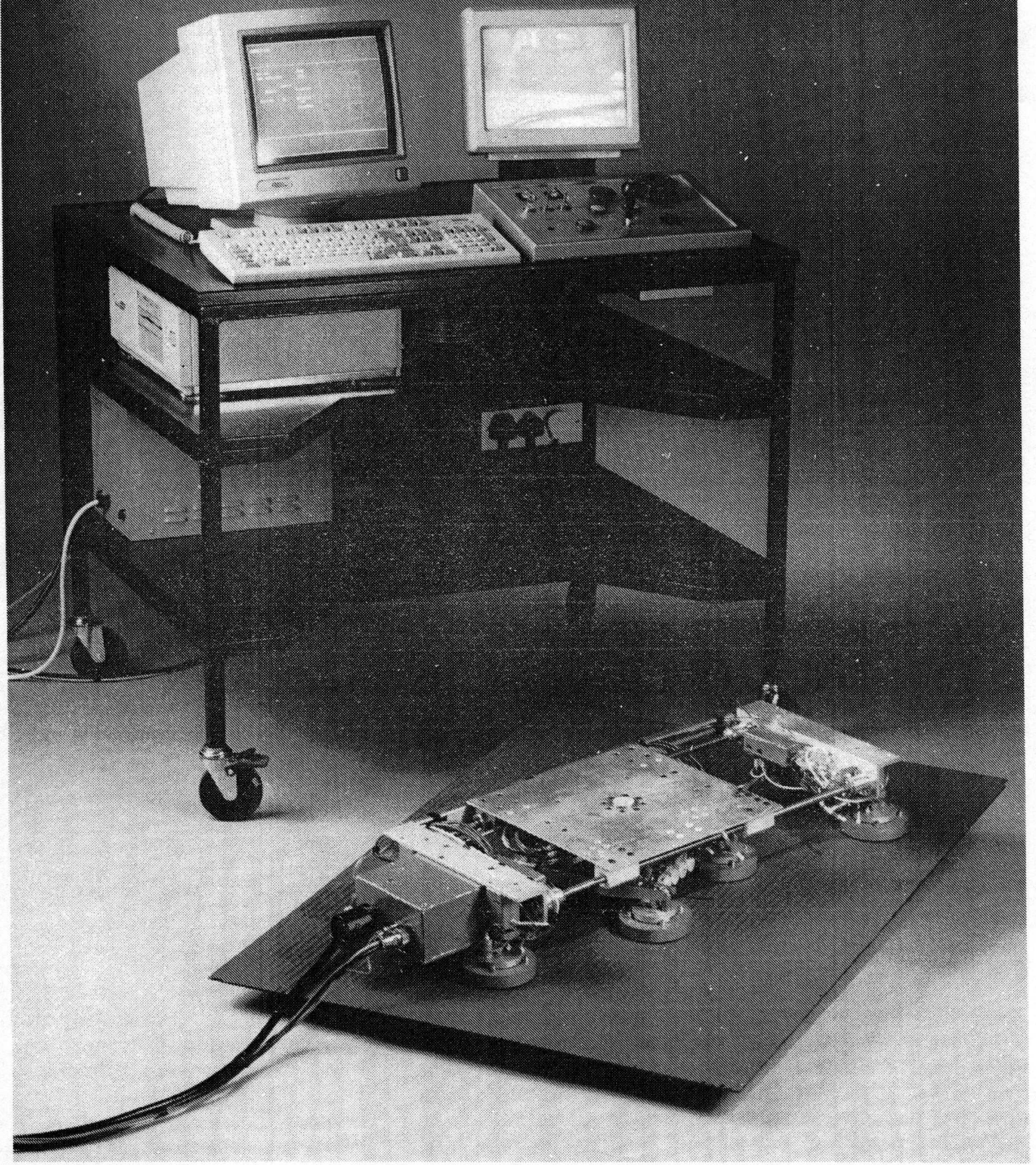

